\title{
THE SHELL OF V603 AqL AND THE EARLY STAGES \\ OF THE NOVA EVENT
}

\author{
H. WEAVER
}

Dept. of Astronomy and Radio Astronomy Laboratory, University of California, Berkeley, Calif., U.S.A.

\section{Introduction}

Nova V603 Aq1 1918 was uniquely suited for studies of the structure of its shell because of the very favorable orientation of the shell in space. Additionally, almost by chance, spectroscopic observations were made in such a way that they permitted derivation of a three dimensional model of the shell.

\section{Symmetry, Anti-Symmetry in the Shell}

V603 Aql, as we see from its light curve (Figure 1), was a typical fast nova which showed strong periodic fluctuations in light during the transition stage. The period of fluctuation was approximately 10 days, during which time the visible light changed by roughly $50 \%$.

Approximately three and one half months after maximum light, Barnard (1919, 1920) observed that the nova was surrounded by a nebular shell which was later found to be expanding at the rate of $2^{\prime \prime} \mathrm{yr}^{-1}$. Thirteen and one-helf months after maximum, when the nebular shell was $2.2^{\prime \prime}$ in diameter, Wright (1919) used a slitless

MAGNITUDE 2

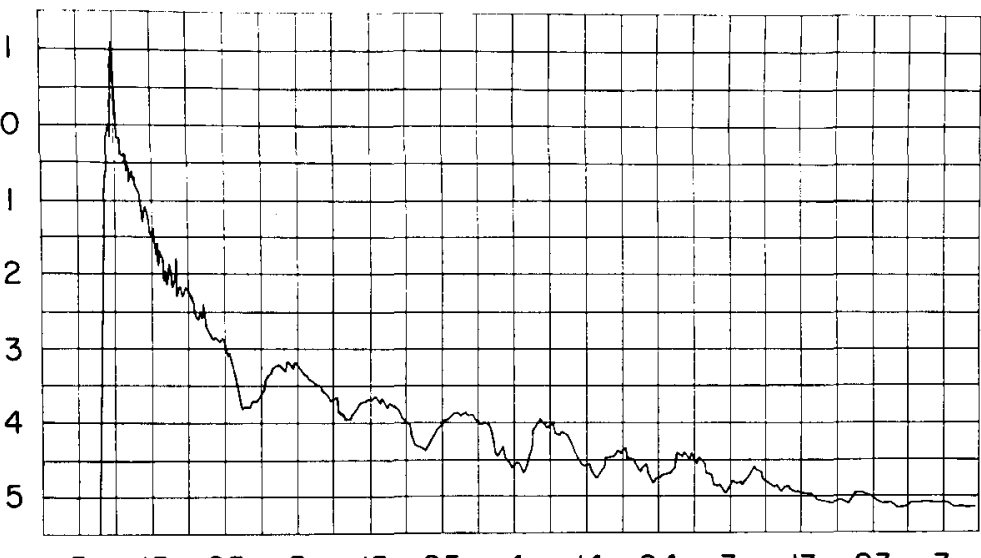

1918 JUNE

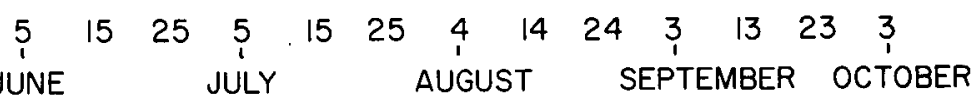

Fig. 1. The light curve of V603 Aql. Data are from Leon Campbell, Ann. Harv. Coll. Obs. 81, 179, 1920. 


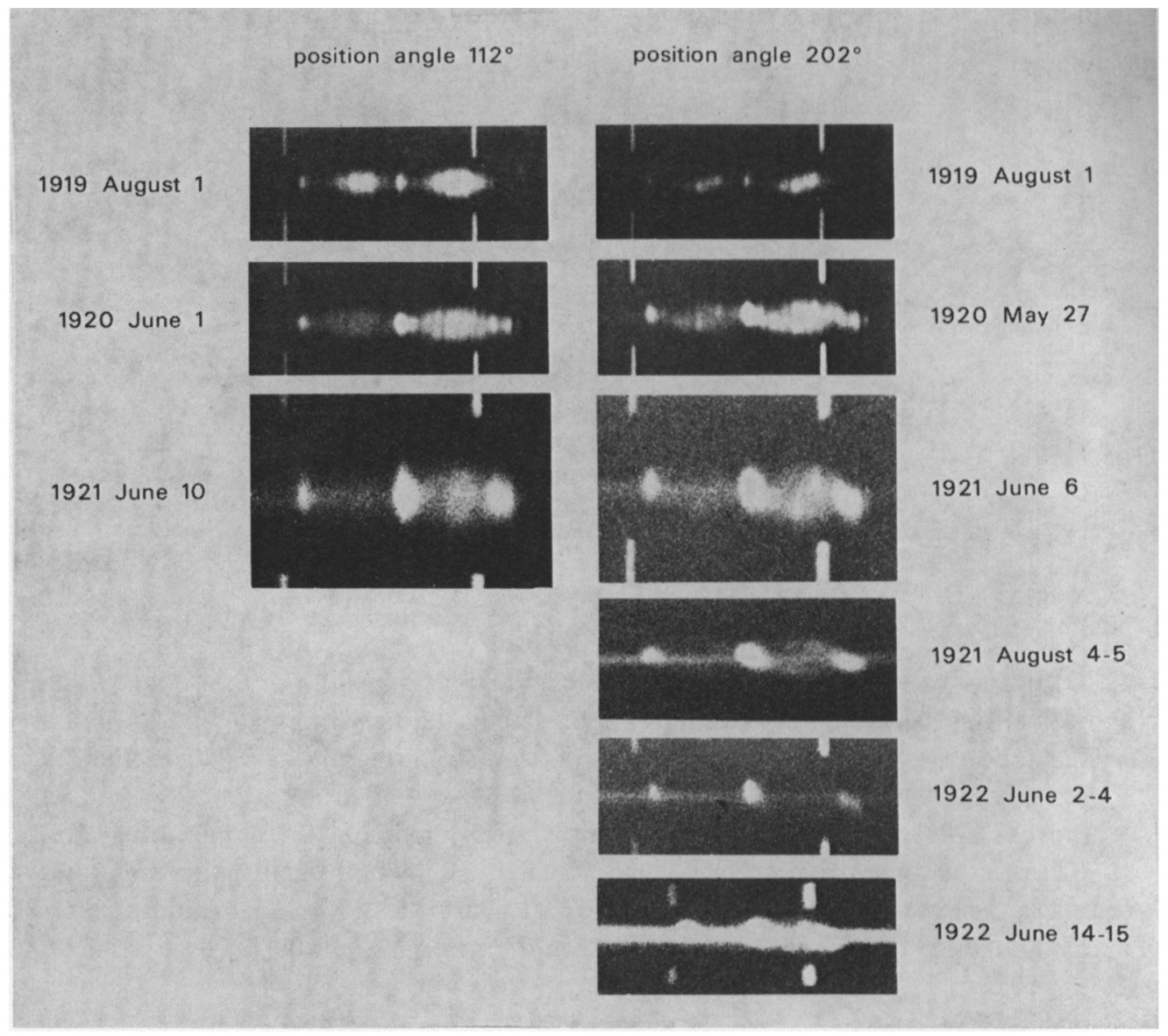

Fig. 2. The spectral development of V603 Aql during 1919-1922. The lines shown in each spectrogram are the $\mathrm{N} 1$ and $\mathrm{N} 2$ lines of [O III].

spectrograph to observe the $\mathrm{N} 1$ and $\mathrm{N} 2$ lines of [O III] originating in the shell. From a series of exposures made by rotating the spectrograph so that the refracting edge of the prism occupied different position angles across the shell, he demonstrated that the visible expanding shell was not spherically symmetrical. In position angle $112^{\circ}$ the spectrographic images were symmetric; in position angle $202^{\circ}, 90^{\circ}$ different from the first, the spectrographic images were antisymmetric. Following Wright's discovery, Lick spectrograms of the nova were normally taken with the slit in one of these two position angles, and the nebular image was held stationary on the slit.

The spectral development of the nova shell during the period 1919-1922 is shown in Figure 2. In any one position angle the essential nature of the slit-spectrographic image of the shell, which was always complex in character, remained remarkably constant in form over the years. Change in any one feature within either the N1 or N2 line involved intensity and vertical extent of the feature - that is, expansion 
of the shell - but not velocity, which remained constant for any feature within a line. A high degree of symmetry, antisymmetry remained at all times the outstanding characteristic of the structure.

\section{The Form of the Shell Produced by Different Types of Ejection}

A shell of gas producing slit-spectrographic images having regularities as pronounced as those shown by V603 Aql must be a highly organized structure of complex geometrical form. To gain insight into the nature of slit-spectrographic images produced by shells of various geometrical forms we look at the results from a series of model calculations.

Figure $3 a$ illustrates what we would see if we examined a completely filled, uniformly expanding spherical shell of optically thin gas with a slit spectrograph. The slit is assumed to be placed in each of three representative position angles, one after the other.

In Figures $3 b$ through $3 \mathrm{~h}$ we see slit-spectrographic images for an infinitely thin, uniformly expanding shell, a series of uniformly expanding gaseous rings, and a series of uniformly expanding cones. In the latter two cases the axis of the system is shown in three different positions; in every case the gas is assumed to be optically thin.

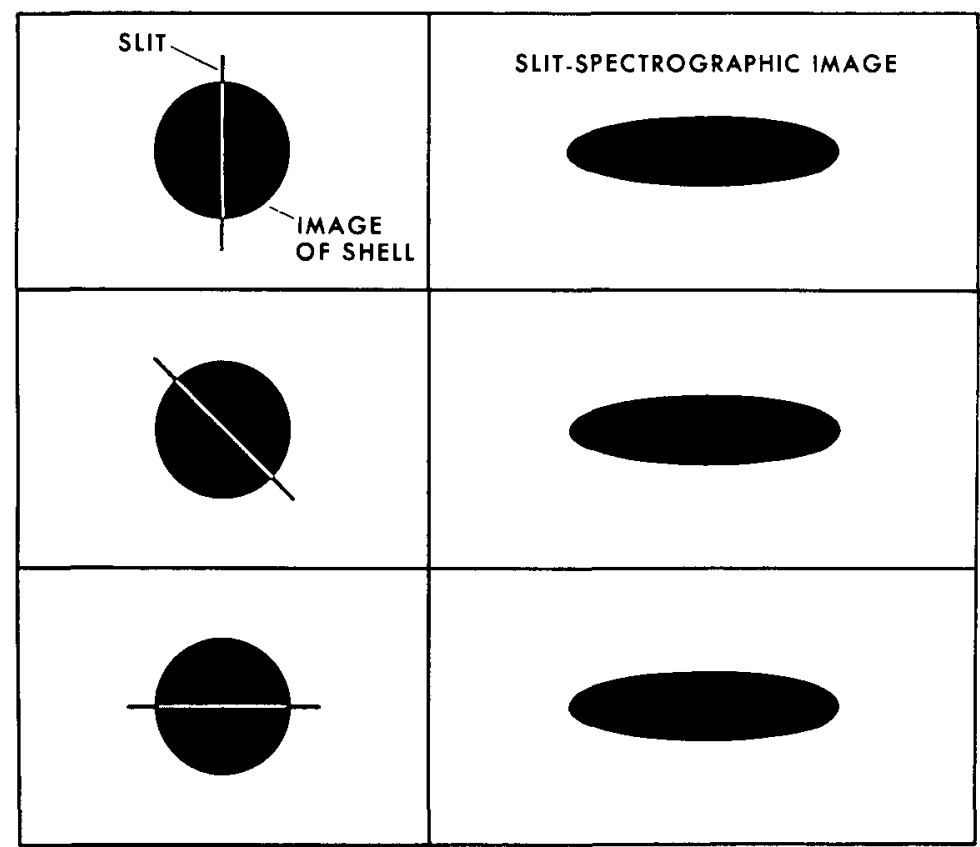

Fig. 3a.

Figs. 3a-h. Results for model calculation of slit-spectrographic images arising from various types of gas ejection. The velocity of ejection has always been taken to be isotropic. The gas is optically thin. (a) A completely filled sphere of gas. (b) An infinitely thin spherical shell of gas. (c, d, e) Rings of gas. The axis of the ring system is shown at three different tilt angles with respect to the line of sight. (f, $g, h$ ) Cones of gas. The axis of the cone system is shown at three different tilt angles with respect to the line of sight. 


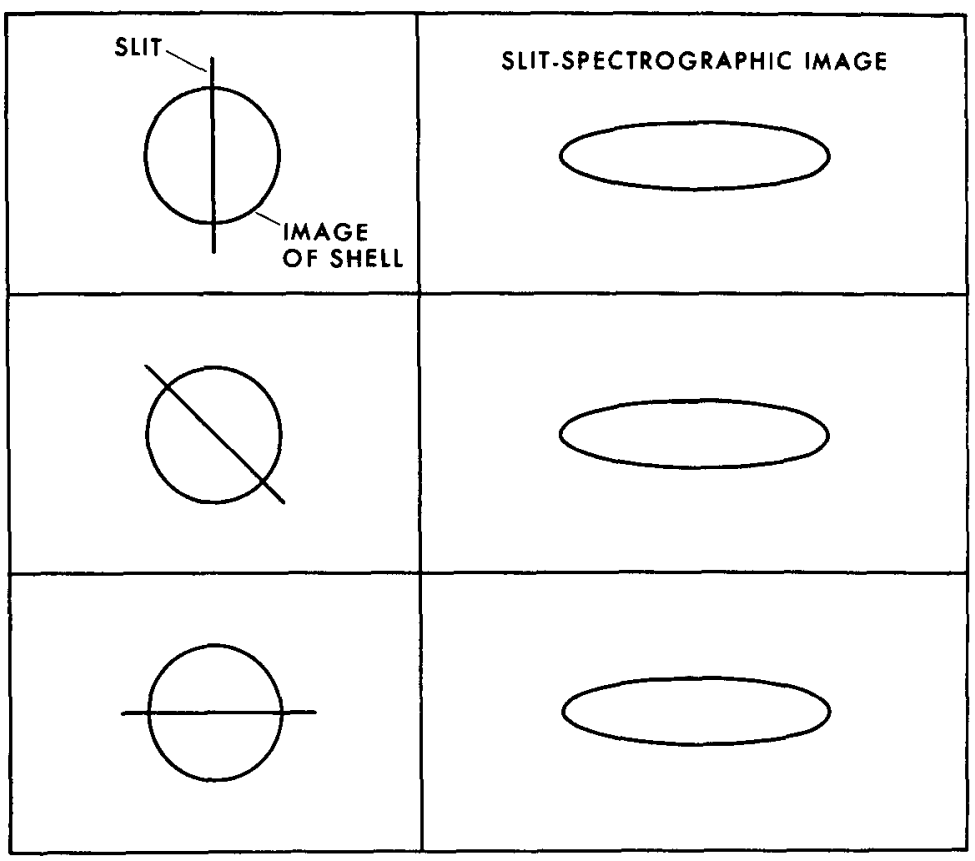

Fig. 3b.

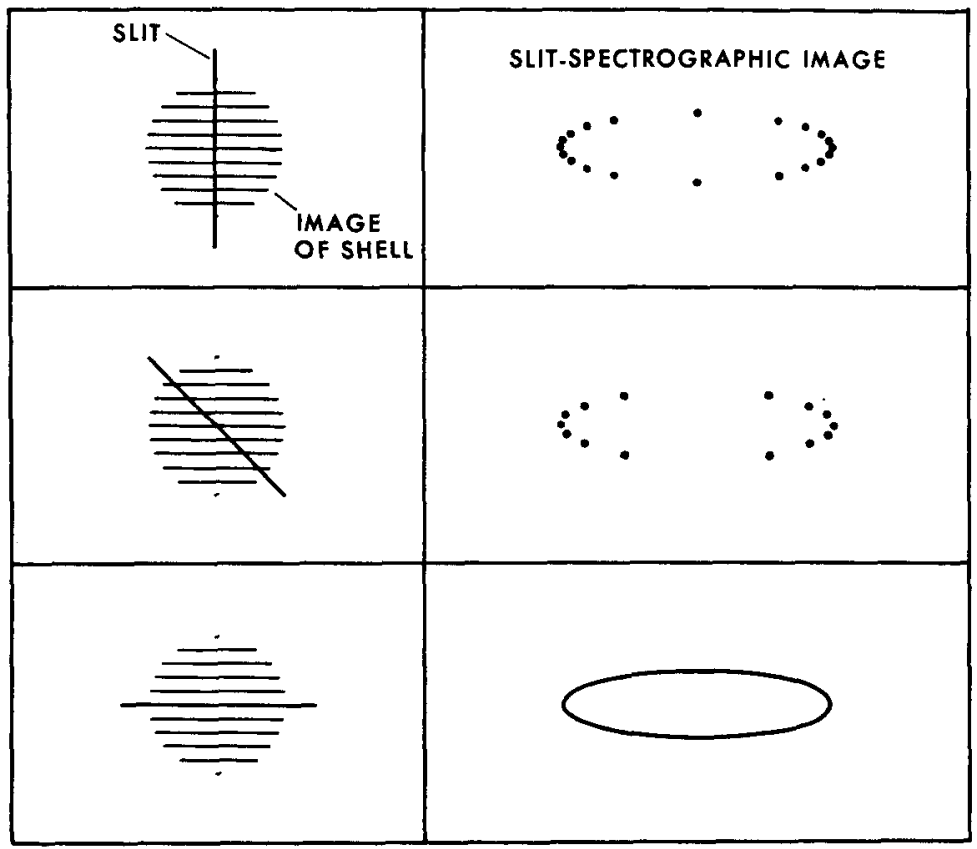

Fig. 3c. 


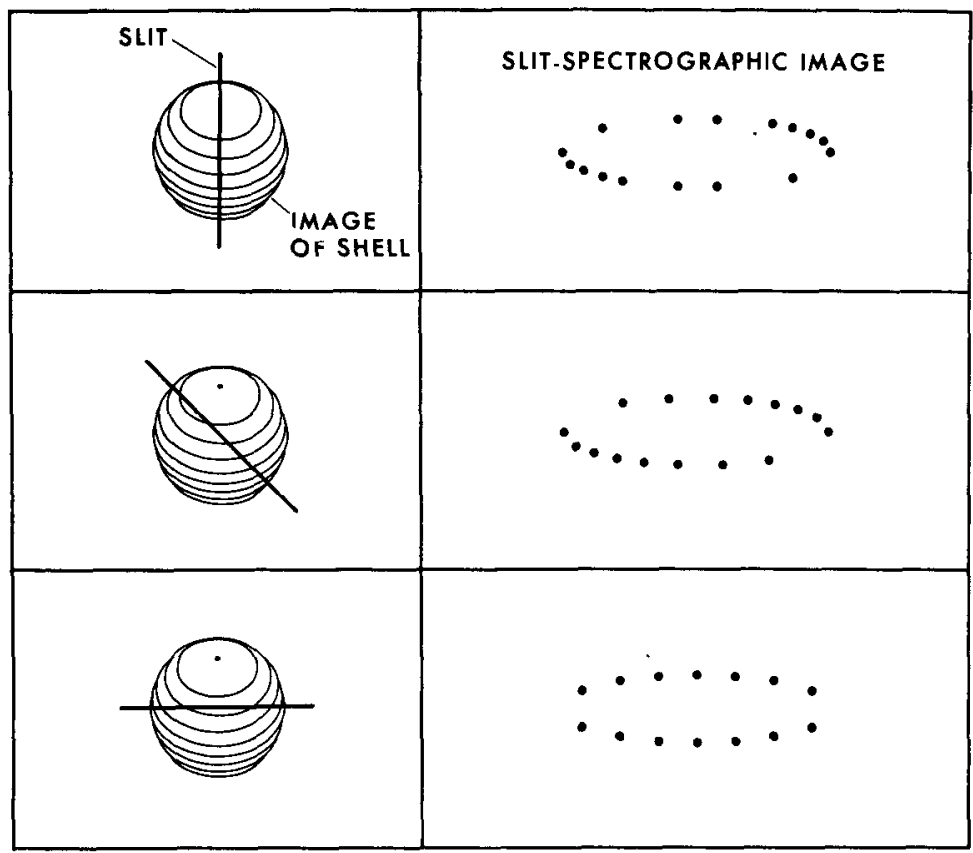

Fig. 3d.

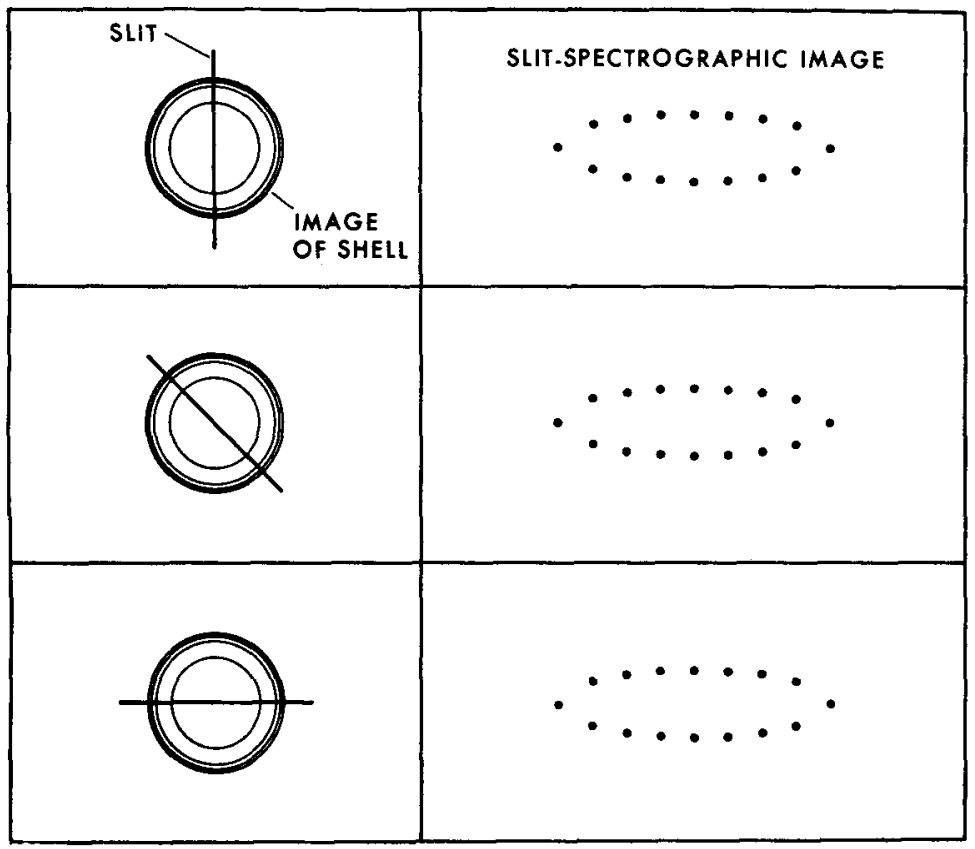

Fig. 3e. 


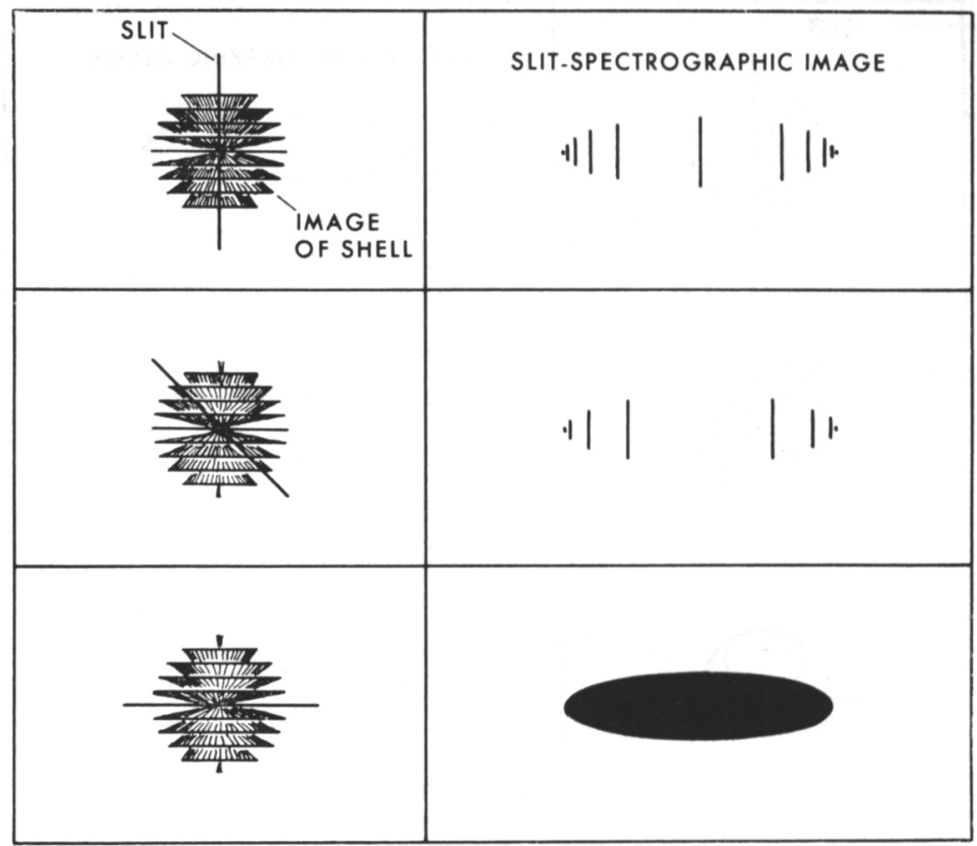

Fig. 3f.

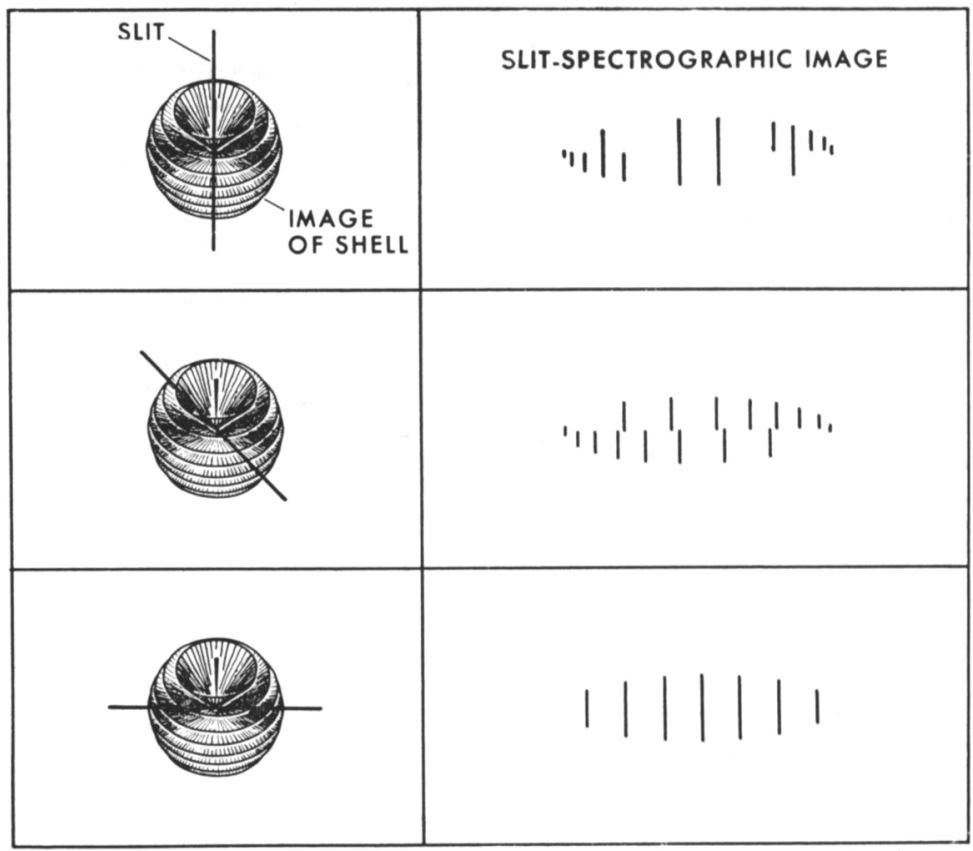

Fig. 3g. 


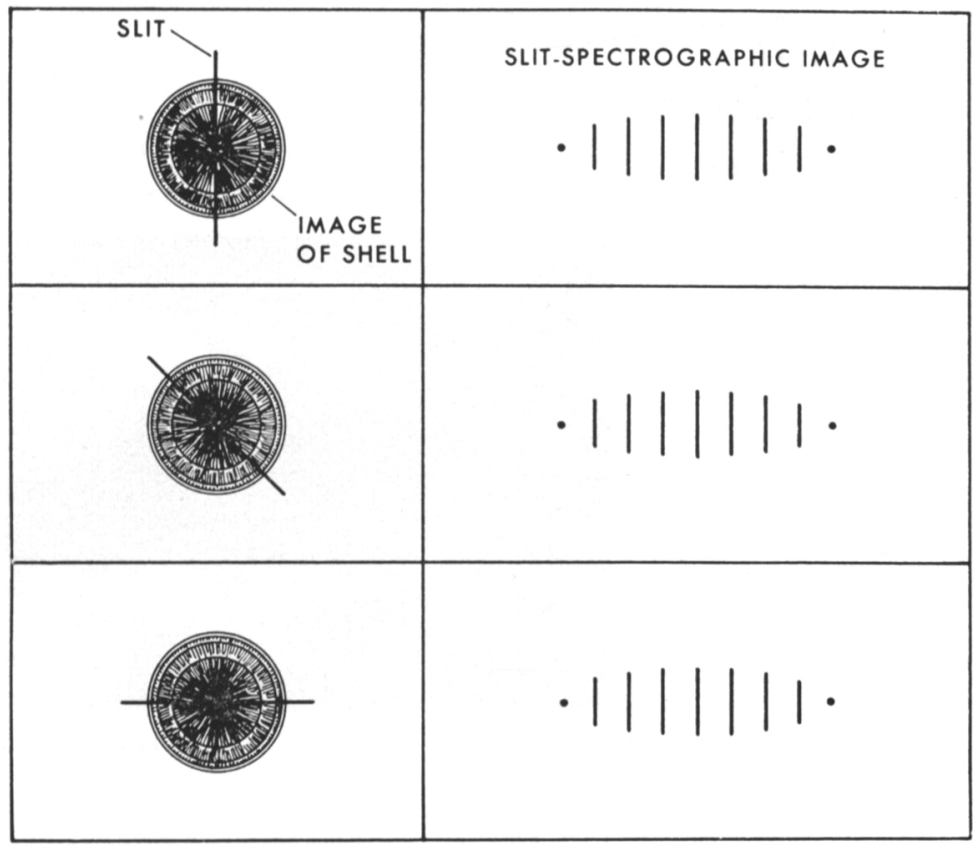

Fig. 3h.

All of the model calculations displayed in Figure 3 have been made for an isotropically expanding shell. If non-isotropic expansion were to take place so that an ellipsoidal shell would be produced rather than a spherical one, there would be no change in the character of the slit-spectrographic images pictured in Figure 3; they would simply be transformed by smooth stretching in the slit-spectrographic image plane.

\section{Reconstruction of the Shell of V603 Aql}

We can invert the problem illustrated in Figure 3. If for a real nova shell we have slit-spectrographic images taken with the slit in a number of different position angles, we can reconstruct the form of the expanding envelope provided:

(a) that all features originate at the same time, and

(b) that the motion of the gas giving rise to a specific feature in the line is rectilinear.

In the case of V603 Aql these requirements are met. We shall also assume that the expansion velocity is isotropic. This assumption is probably not correct for reasons to be mentioned later. Failure of the assumption means only that the reconstructed picture of the nova shell will be somewhat distorted in different directions, but not changed in character.

Figure 4 shows examples of slit-spectrographic images taken in 1919, approximately 15 months after maximum light. The lines shown are $\mathrm{N} 1$ and $\mathrm{N} 2$ of [O III]. Figure 5 shows slit-spectrographic images from the same period but at higher dispersion, approximately $10 \AA \mathrm{mm}^{-1}$. From these and other slit-spectrographic images, the cross 


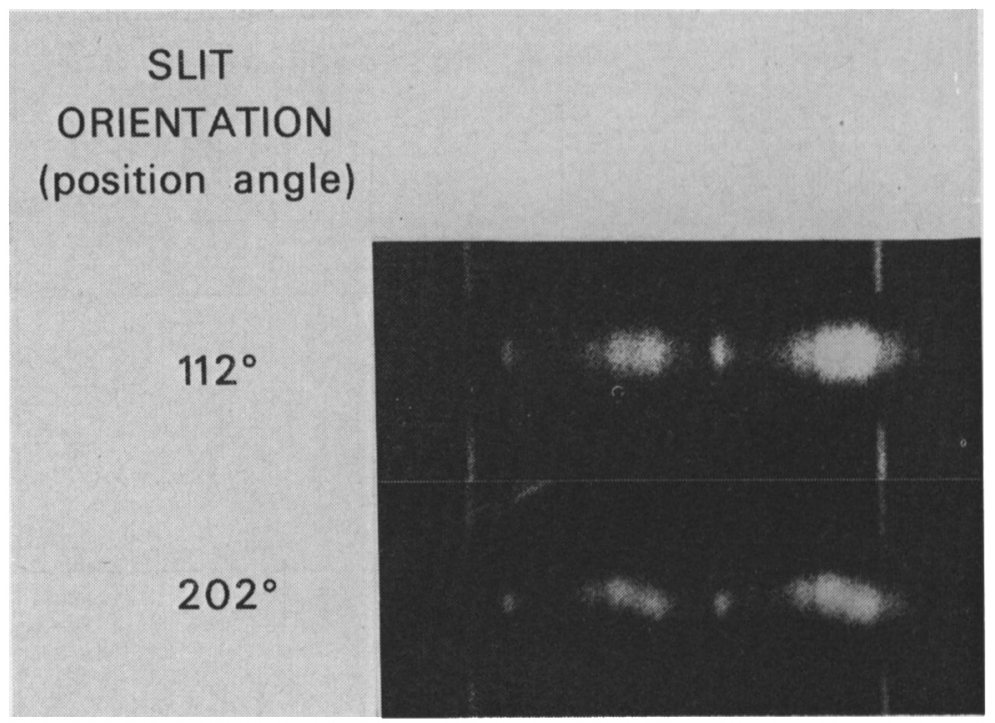

Fig. 4. Slit spectrographic images of V603 Aql taken in 1919. The lines shown are N1 and $\mathrm{N} 2$ of [O $\mathrm{III}]$.

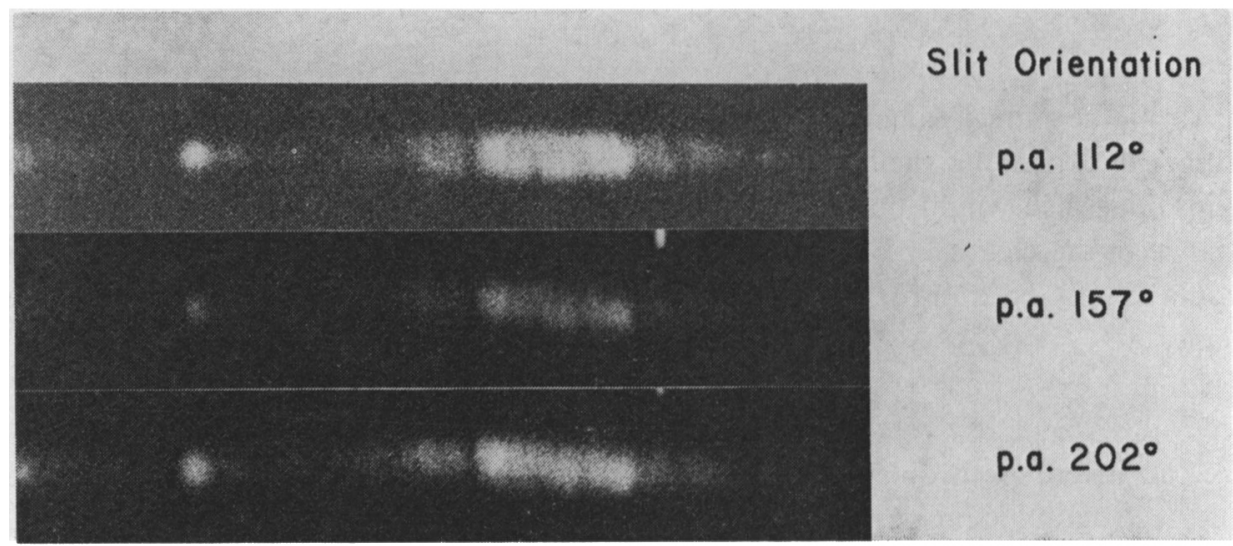

Fig. 5. Slit spectrographic images of V603 Aql taken in 1919; dispersion approximately $10 \AA \mathrm{mm}^{-1}$. The line shown is $\mathrm{N} 1$ of [O III].

section of the nova shell was reconstructed for the symmetrical, $112^{\circ}$, position angle as shown in Figure 6a. The shell thickness on this and the drawings that follow must be regarded as schematic only, and is shown greater than it was in reality.

Figure $6 \mathrm{~b}$ shows the reconstruction of the antisymmetric, $202^{\circ}$ position angle cross section of the shell. It is particularly notable that this cross section has two diametrically opposite holes in it. These holes, combined with the particular skewed intensity pattern present in the shell in 1919 , produced the antisymmetry observed by Wright in position angle $202^{\circ}$.

Figure 7 is a drawing of the reconstructed three-dimensional model of V603 Aql. 
The shell is composed of truncated coaxial cones. That is, in 3-dimensional space the trajectory of each mass of gas in the shell is along a radius drawn outward from the nova. Motion along this radial trajectory remains constant in direction in space and in velocity, hence the observed radial velocity of any feature in the slit-spectrographic image remains constant in time.

Some cones are intense emitters because of their density and excitation, others are only very weak emitters. The very weakly emitting cones are the source of the fine dark 'lines' that cross the slit-spectrographic image of the nova. These dark 'lines' are not absorption features. The optical depth is $\ll 10^{-5}$ in the [O III] lines in which the observations were made.

The shell is distorted as is readily seen on the drawing. It bulges outward in the general regions of the holes that are present in the shell.

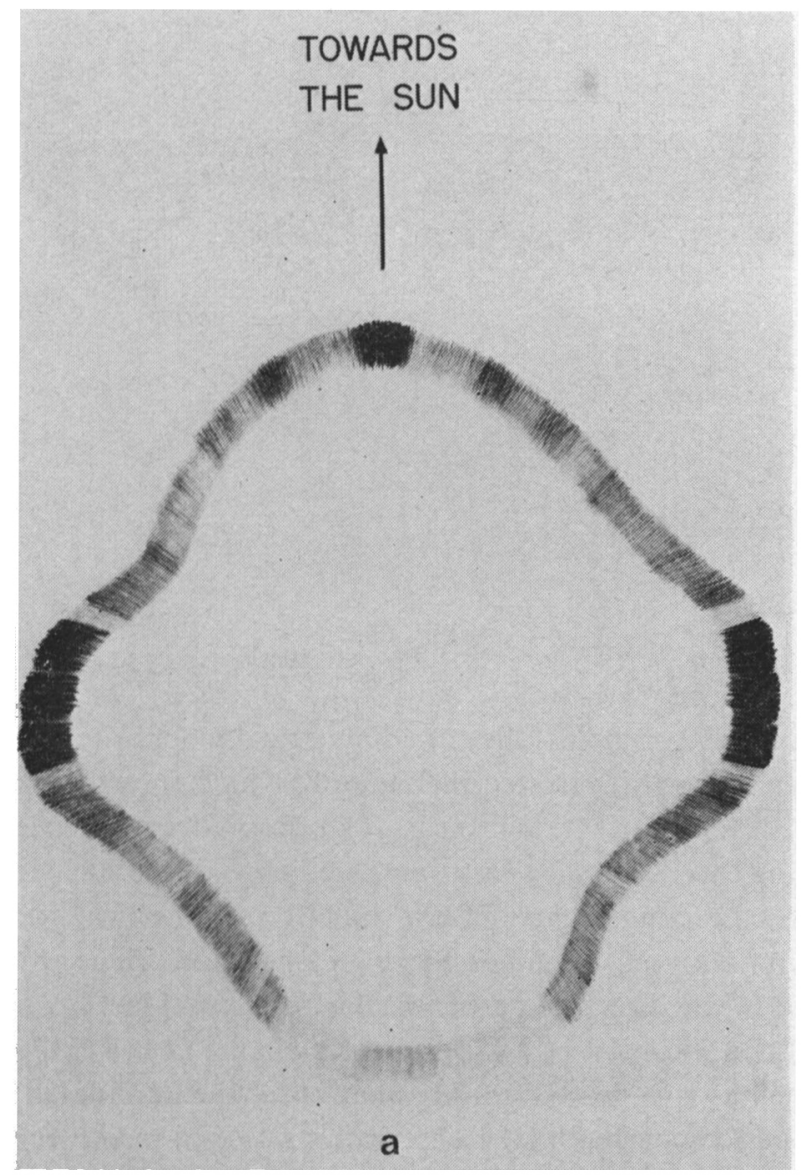

Fig. 6a-b. (a) Drawing of the cross section of the shell of V603 Aql observed when the slit of the spectrograph was in p.a. $112^{\circ}$. Intensity distribution in the shell is shown as it was in 1919. (b) Drawing of the cross section of the shell of V603 Aql observed when the slit of the spectrograph was in p.a. $202^{\circ}$. Intensity distribution in the shell is shown as it was in 1919. 


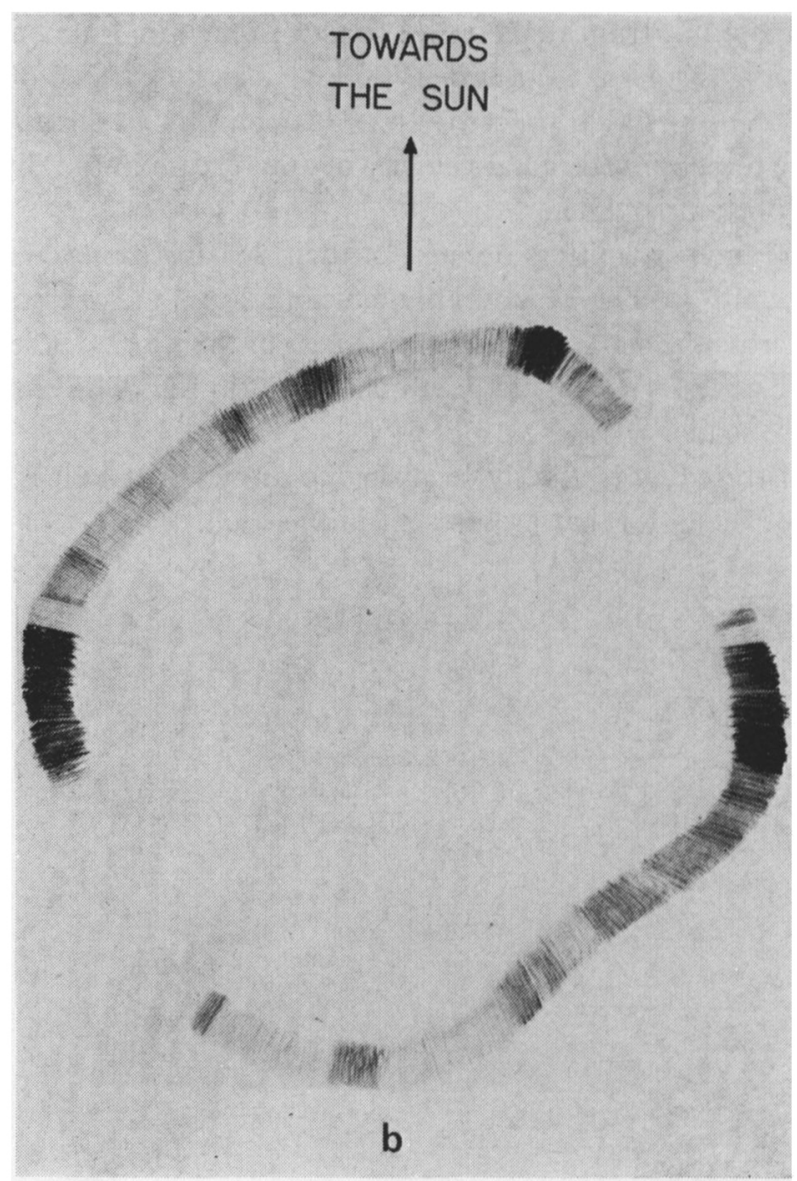

Fig. 6b.

There are, of course, two holes; they are diametrically opposite to each other in the shell. These holes are well illustrated in Figure 8, which shows a slit-spectrographic image of V603 Aq1 taken at Mt. Wilson in 1920. Radially symmetrical breaks in the quasi elliptical ring that is the slit-spectrographic image of the shell are clearly visible. These breaks are the counterparts of the radially symmetrical holes in the shell. Angular resolution was very high for the plate shown in Figure 8, hence the shell was well resolved in the direction perpendicular to dispersion.

The picture of the shell of V603 Aql shown in Figure 7, it should be emphasized, represents the shell as it appeared in the radiation of the N1 line of [O III].

Over the time period covered by the observations used in reconstruction of the shell no velocity changes were detected in any parts of the shell. Only changes in angular diameter of the shell and in the relative brightnesses of the truncated cones were observed to take place. The brightness changes observed over the period 1919-1922 follow the decay times of [O $\mathrm{III}]$ under conditions of different electron density. 


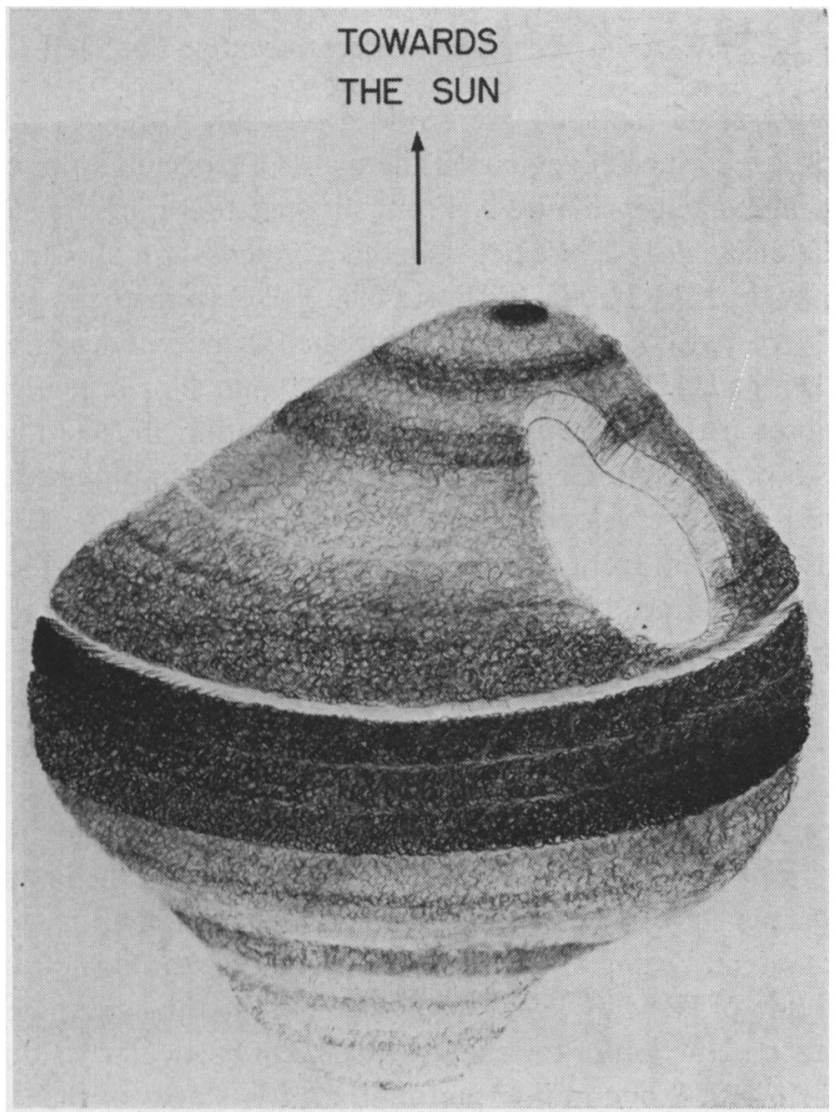

Fig. 7. Drawing of a three dimensional model of V603 Aql derived from spectrograms taken at the Lick Observatory in 1919.

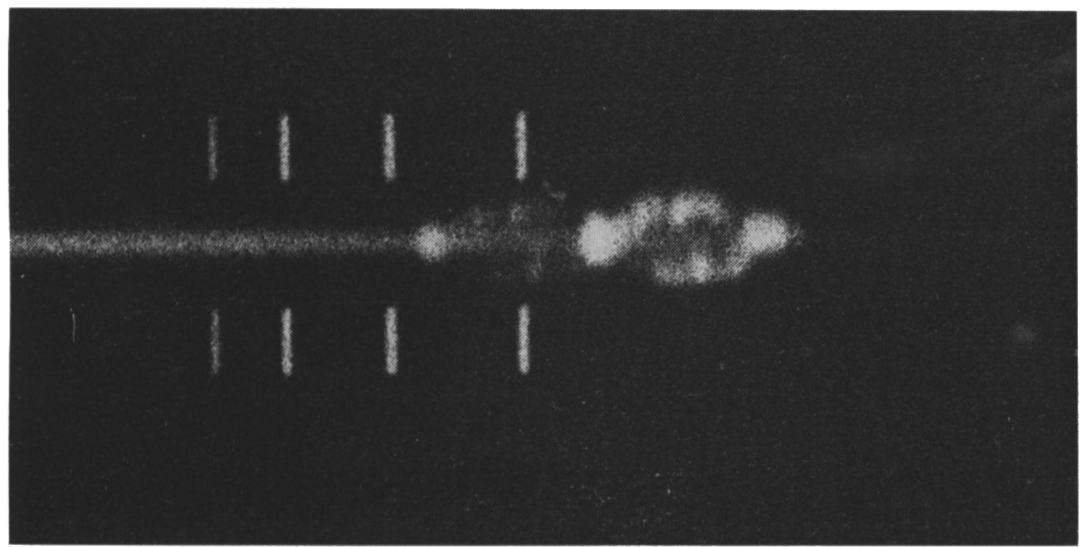

Fig. 8. Slit-spectrographic image of V603 Aql taken at Mt. Wilson on July 23, 1920. The slit was in p.a. $90^{\circ}$. (I am indebted to Dr R. Minkowski for this photograph.) 


\section{Tests of the Technique of Reconstructing the Shell}

(a) Having reconstructed the shell, we are in a position to relate features in a slitspectrographic image to specific regions of the shell. Of particular interest is the bright poleward spot marked out in Figure 9. From slit-spectrographic images with the slit in different position angles we predict that this condensation should appear on the sky in position angle $22^{\circ} \pm 3^{\circ}$, at a distance $0.42 \pm 0.02$ from the center towards the edge of the shell. We here count the radius of the shell as unity. On a direct photograph of V603 Aql taken at Mt. Wilson by Baade in 1940 and shown in Figure 10, we see the shell and observe that there is a bright condensation in position angle $28^{\circ}$ at distance 0.43 . This condensation, visible on the direct photograph, produced the bright feature of highest negative velocity seen in the spectra taken $20-22 \mathrm{yr}$ earlier. The plate taken in 1950 suffers from poor seeing. It is not certain that the condensation was visible at that time. Since 1950 both shell and condensation have faded and cannot now be photographed.

(b) We consider next the tilt angle of the axis of the cone system with respect to the line of sight. In Figure 11 we see a collection of Lick spectrograms of relatively high dispersion $\left(10 \AA \mathrm{mm}^{-1}\right)$ taken in a number of different position angles. Such a set of spectrograms permits determination of the cone axis with high accuracy. The fact that the major narrow dark 'lines' and other features cross the images at the same velocity whatever the slit orientation means that the line of sight is very close to the axis of the cone system. Detailed measurements show that the axis of the cone system differs from the line of sight by less than a degree. It is this fortunate circumstance that makes the reconstruction of the shell of V603 Aql particularly simple and makes V603 a kind of Rosetta Stone in interpreting the early stages in the development of novae. Because of the near perfect alignment of the axis of the cone system and the line of sight, we can specify what part of the shell was involved in producing each portion of a spectral line even when the image was trailed along the slit as it was during the early observations of the nova.

\section{Early Spectral Changes in the Nova Spectrum and Their Relationship to the Shell}

I wish next to trace the early spectral stages of the nova and show how they relate to the light curve (Figure 1) and the three-dimensional model of the nova just described (Figure 7). The points to be emphasized in this discussion are as follows:

(a) The shell developed in a very short time at the very start of the nova process.

(b) The shell underwent acceleration, particularly near the time of maximum light.

(c) For only a few days near the time of maximum light and maximum shell acceleration, double absorption lines were present in hydrogen and other elements in the shell.

(d) The lines in the spectrum divide into two quite different velocity structures which we can identify with the shell and with the nova.

(e) A series of complex changes took place in the spectrum when the nova fluctuated 


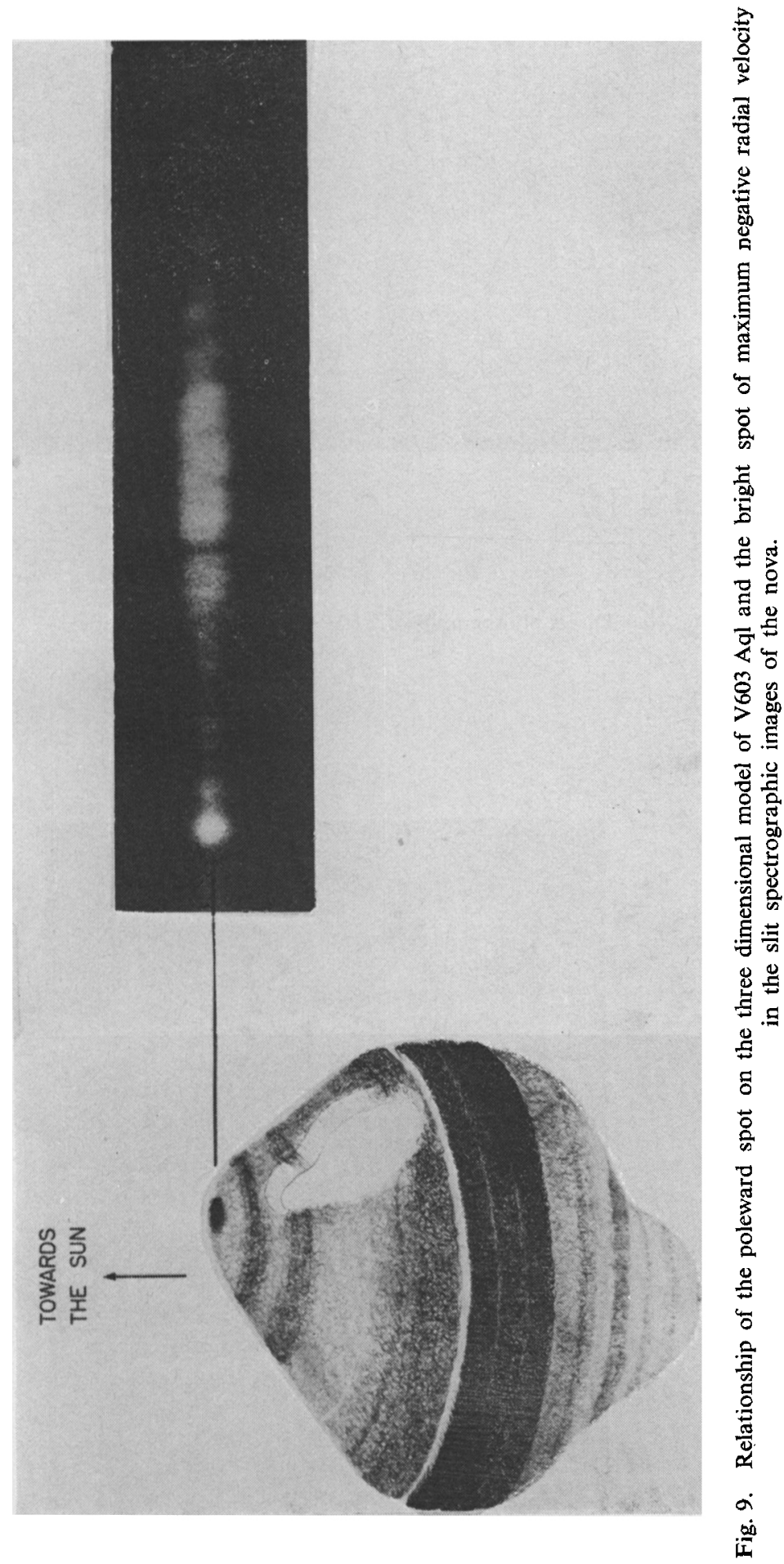




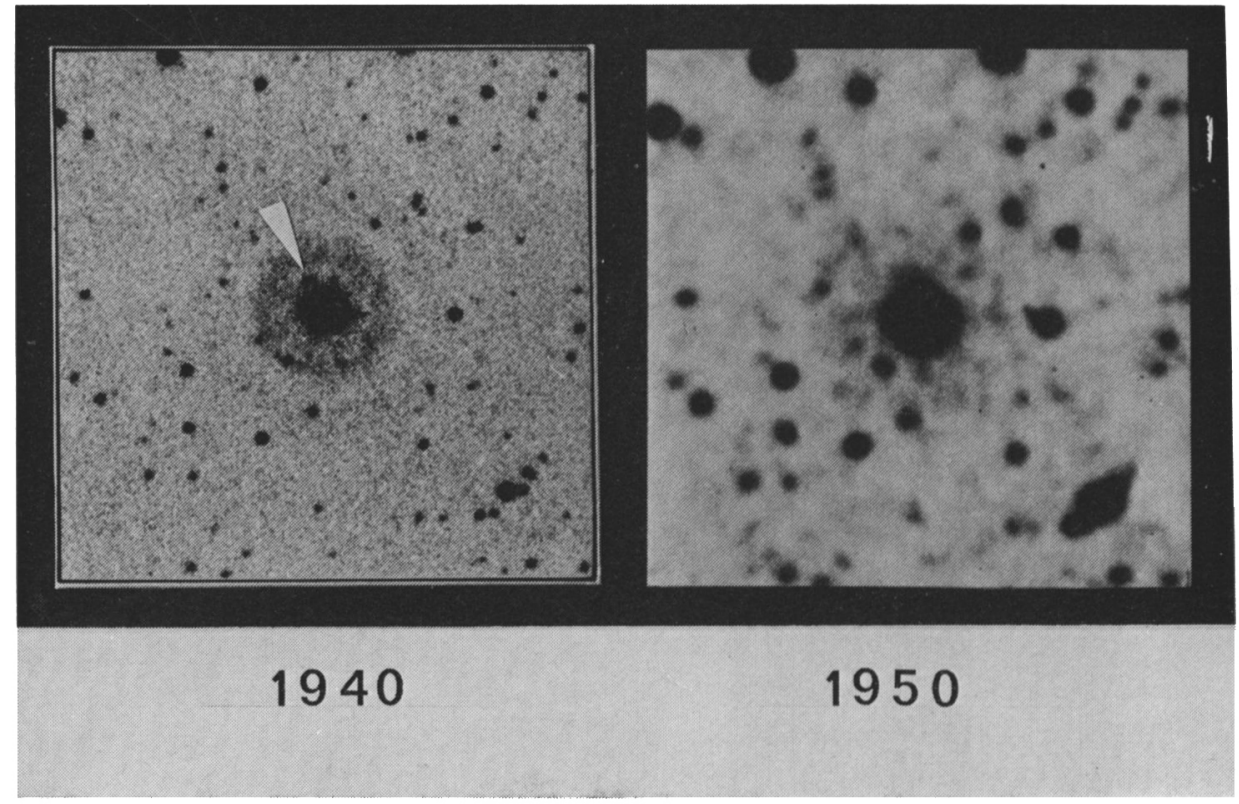

Fig. 10. Direct photographs of V603 Aql taken by W. Baade.

\section{SLIT ORIENTATION}

\section{(position angle)}

$22^{\circ}$

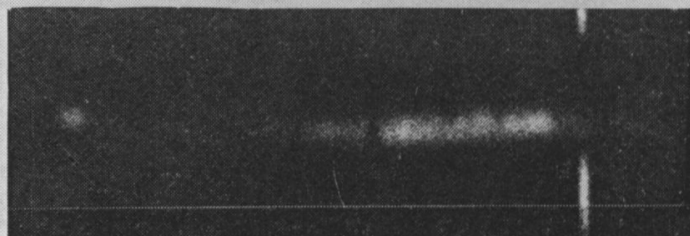

$56^{\circ}$

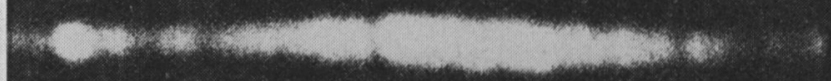

$112^{\circ}$

(1)

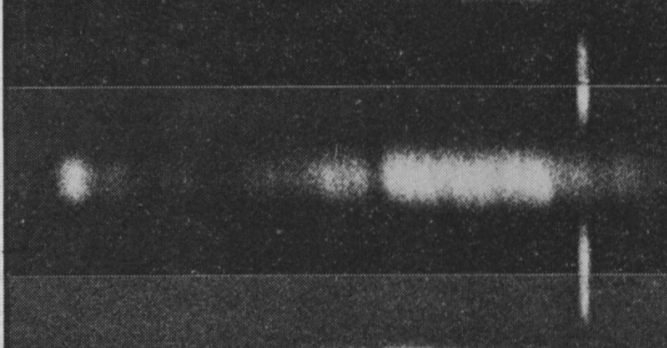

$202^{\circ}$

$293^{\circ}$

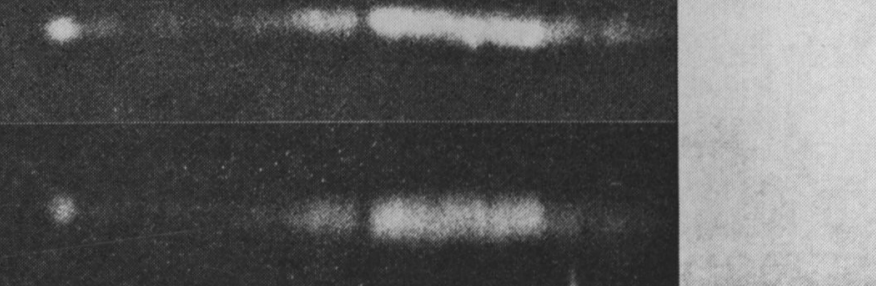

Fig. 11. A series of spectrograms of V603 Aql taken in 1919 with the Mills Spectrograph at a variety of positions angles as indicated. The feature shown is the N1 line of [O III]. 
in light during the transition stage. During these fluctuations the two velocity structures mentioned in (d) were clearly resolved.

Finally, I shall present a physical model of the nova that brings all these phenomena together and shows how the shell shown in Figure 7 was produced.

(i) The first spectrum of the group shown in Figure 12 was taken 0.9 days before maximum light. The nova was then approximately one day old. We see in the spectral range covered only a broad absorption line of $\mathbf{H} \beta$. At the time the spectrum was taken the shell was of the order one astronomical unit in radius and similar in character to the atmosphere of an early supergiant. The opacity of the expanding gas was too high to permit visibility of the internal structural character of the shell.

(ii) By 0.1 to 0.9 days after maximum light, the opacity had decreased enough to permit the internal structure of the shell to be seen. By 0.9 day after maximum the characteristic bright equatorial part of the shell was visible in its counterpart, the bright central part of the spectral line. The equatorial structure of the shell must have been formed at the very onset of shell formation. It could not have been formed, for example, when the shell was 1-2 astronomical units distant from the nova.

Note that at 0.9 days after maximum light a second absorption feature at velocity greater than the shell expansion velocity had developed in the lines of hydrogen and $\mathrm{Fe}$ II.

Figure 13 shows a collection of early Mills spectrograms of V603 from the Lick collection. There are three features to be especially noted on these spectrograms.

(1) Double absorption lines in the shell. By June 10.8, 0.9 day after maximum, two systems of absorptions had developed in hydrogen as well as other elements making up the shell. This was noted earlier on the Mt. Wilson plates. One of the two absorptions was the 'normal' one arising from the most negative velocity of the expanding shell. Although this normal absorption evolved in velocity, (see Section 2 below), its evolution was relatively slow. It was the original feature in the spectrum; it lasted for as long as absorption was visible. The second absorption was transitory; it had a velocity higher than shell expansion velocity. Double lines originating in the shell existed only during the period of time covered by these spectrograms. The absorption feature at higher-than-shell expansion velocity remained visible for a period of 5-6 days; it was accompanied by emission extending out to its maximum velocity. After disappearance of the once-strong second absorption line, some very weak and broad features may have appeared briefly during the time period covered by these spectra, but no activity at velocities higher-than-shell expansion velocity was visible in lines arising from the shell at any later date.

(2) Acceleration of the shell. From the collection of spectra in Figure 12 we see that the shell of V603 Aql underwent acceleration. Acceleration was particularly large during the first few days after maximum light which occurred on June 9.9.

Note the change in radial velocity of the shell between June 10.8 and 11.9. At that time the force giving rise to the acceleration was approximately 3.5 orders of magnitude greater than the force of gravity experienced by the shell. Such force arising from radiation pressure and/or direct gas pressure in the form of gas streams or shells 


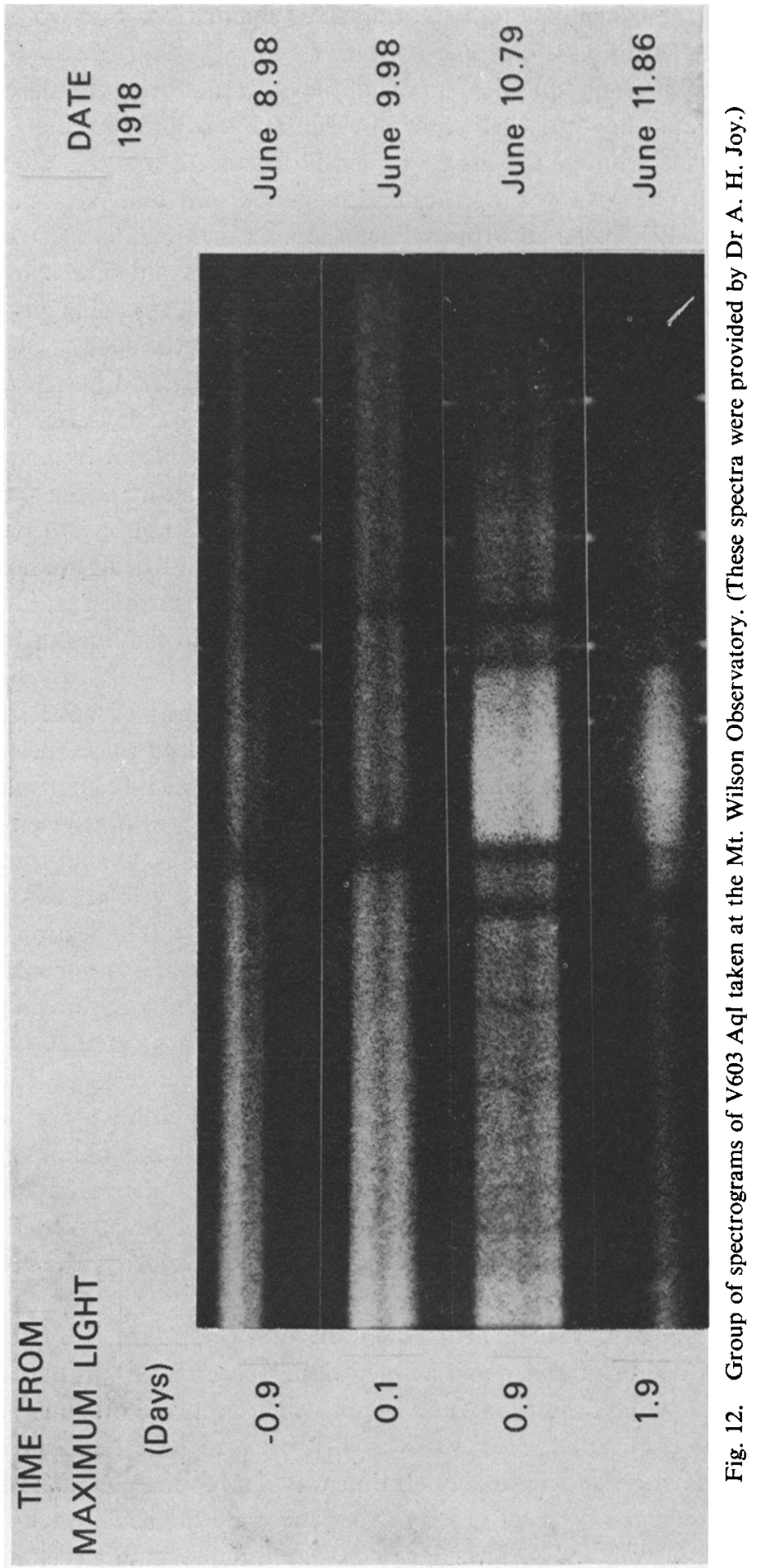




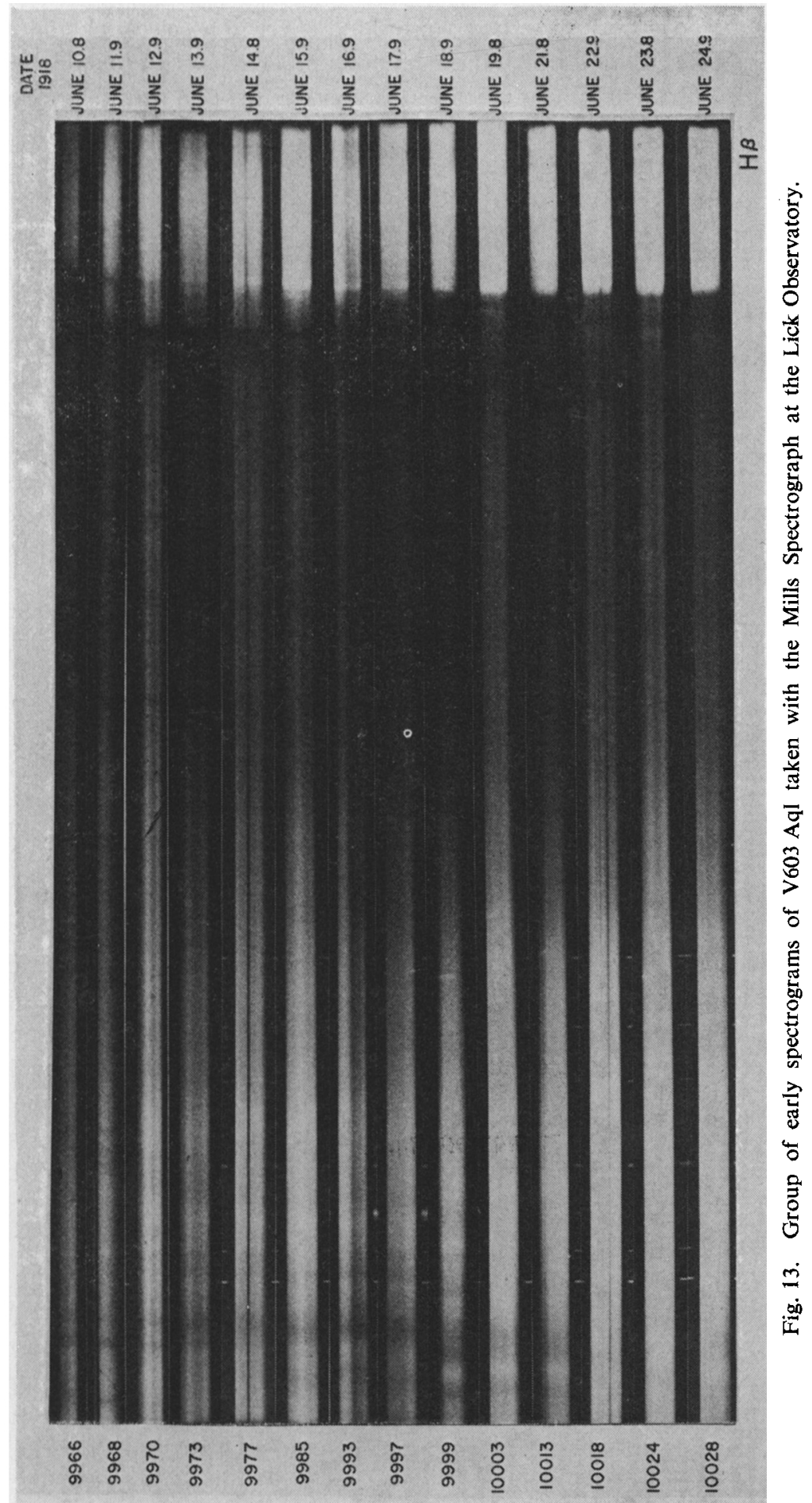


ejected by the nova must have had a profound effect in accelerating and deforming the nova shell. The time at which this maximum pressure was exerted on the shell was also the time - the only time - at which a second absorption feature appeared in the spectral lines originating in the shell. The implication is strong that the shell was partly ruptured by the pressure that caused the acceleration. Rupture of the shell did not take place at the part of the shell pointed directly towards the Sun. There is no velocity, time discontinuity in the negative velocity edge of the hydrogen lines as would necessarily be the case if the shell were disrupted at the point giving rise to maximum negative velocity.

Ejection of gas from other parts of the shell would, by projection, give rise to a mass of gas appearing to move across the shell, away from its center, and producing absorption at a velocity higher than shell expansion velocity while it was between the shell and the observer. Given the velocity of expansion of the blown-out gas, one computes that the blown-out gas should remain visible as absorption for 2-5 days depending upon location and size of the hole blown out of the shell. The computed time is closely similar to the length of time the second hydrogen absorption feature was actually visible.

(3) The existence of two classes of lines in the spectrum.

(a) There are lines with well-defined edges and absorptions on the negative velocity side. Such lines define the shell expansion velocity. Hydrogen is the most prominent example.

(b) There are lines without defined edges and without absorption components on the negative-velocity side. These ill-defined lines show velocities much greater than shell expansion velocity. He II at $\lambda 4686$ and $\mathrm{N}$ III at $\lambda 4640$ are the most prominent lines in this category.

The 4686 and 4640 lines, the principal contributors to the extensive hazy feature on the left-hand side of the spectra in Figure 13, are extremely broad. They show no definite edges; they simply fade out. No absorption appears on the shortward side of the line. These very broad edgeless features are directly related to what has, in the literature, been termed 'Nitrogen Flaring'. The term is somewhat of a misnomer. The effect is not confined to nitrogen. $\mathrm{He}, \mathrm{O}, \mathrm{Fe}$, and other elements are involved also. $\mathrm{He}$ and $\mathrm{N}$ are simply the elements with the most prominent lines showing the phenomenon. The effects might more appropriately be called simply 'flaring'. Flaring was conspicuous during the time period covered by these spectrograms; it was not present at all times. Flaring lines showed a totally different velocity structure from hydrogen, for example, which never flared.

As we shall see with increasing clarity as we look at the data in more detail, a nova spectrum consists of two sets of lines normally present at the same time. One set arises from the expanding shell, the other set arises from ejecta from the nova. These two objects, the shell and the nova, have quite different chemical compositions. The shell is essentially normal in chemical abundances. The nova is a hydrogenexhausted white-dwarf-like object. The ejecta contain no hydrogen, only $\mathrm{He}, \mathrm{N}, \mathrm{O}, \ldots$ and other elements characteristic of a hydrogen exhausted object. This chemical 


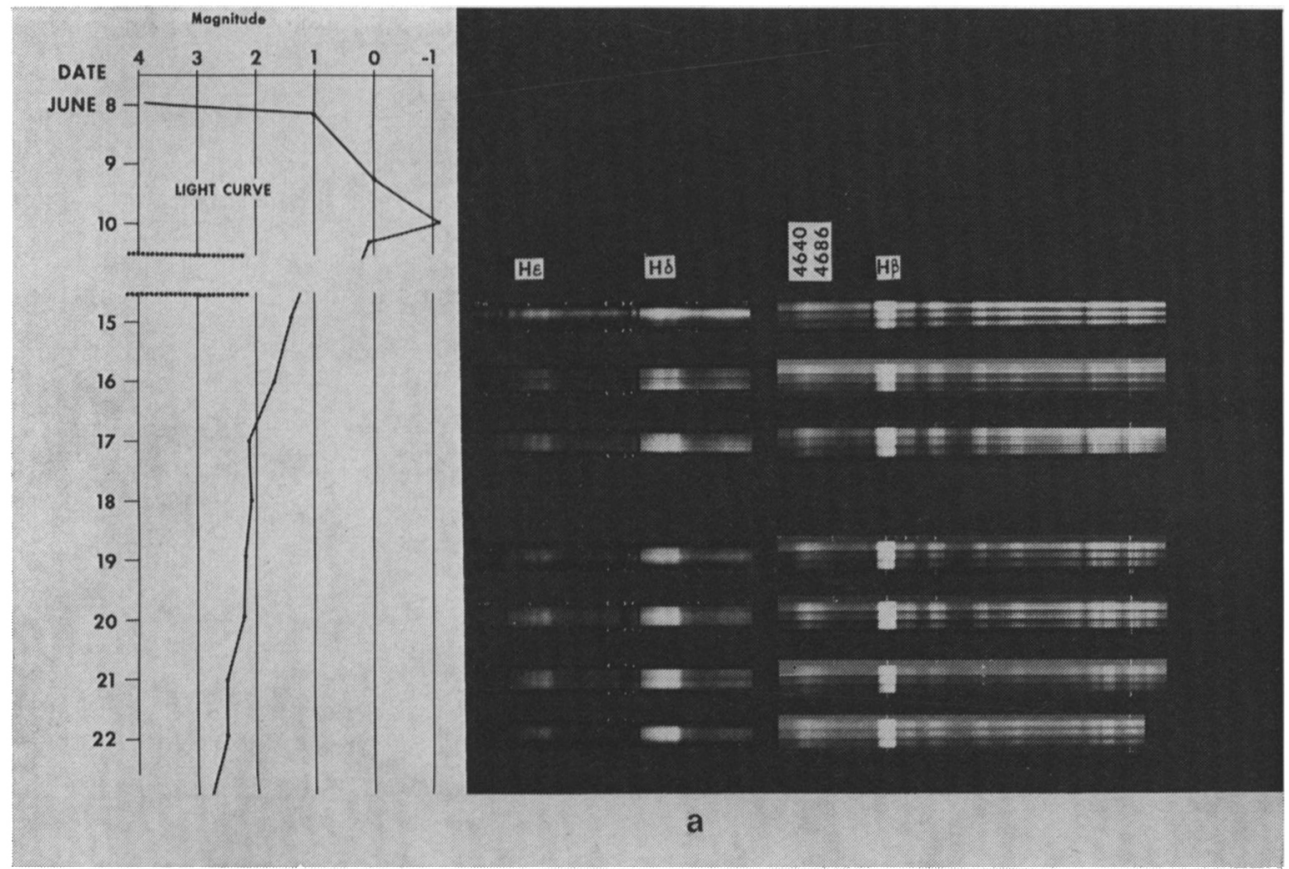

Fig. 14. (a, b) Spectra of V603 Aql taken at the Lick Observatory. The spectrograms showing the red end of the spectrum have been widened by joining three prints of slightly different exposures. The purpose in doing this was to produce prints of sufficient width and of appropriate density to show the spectral lines adequately.

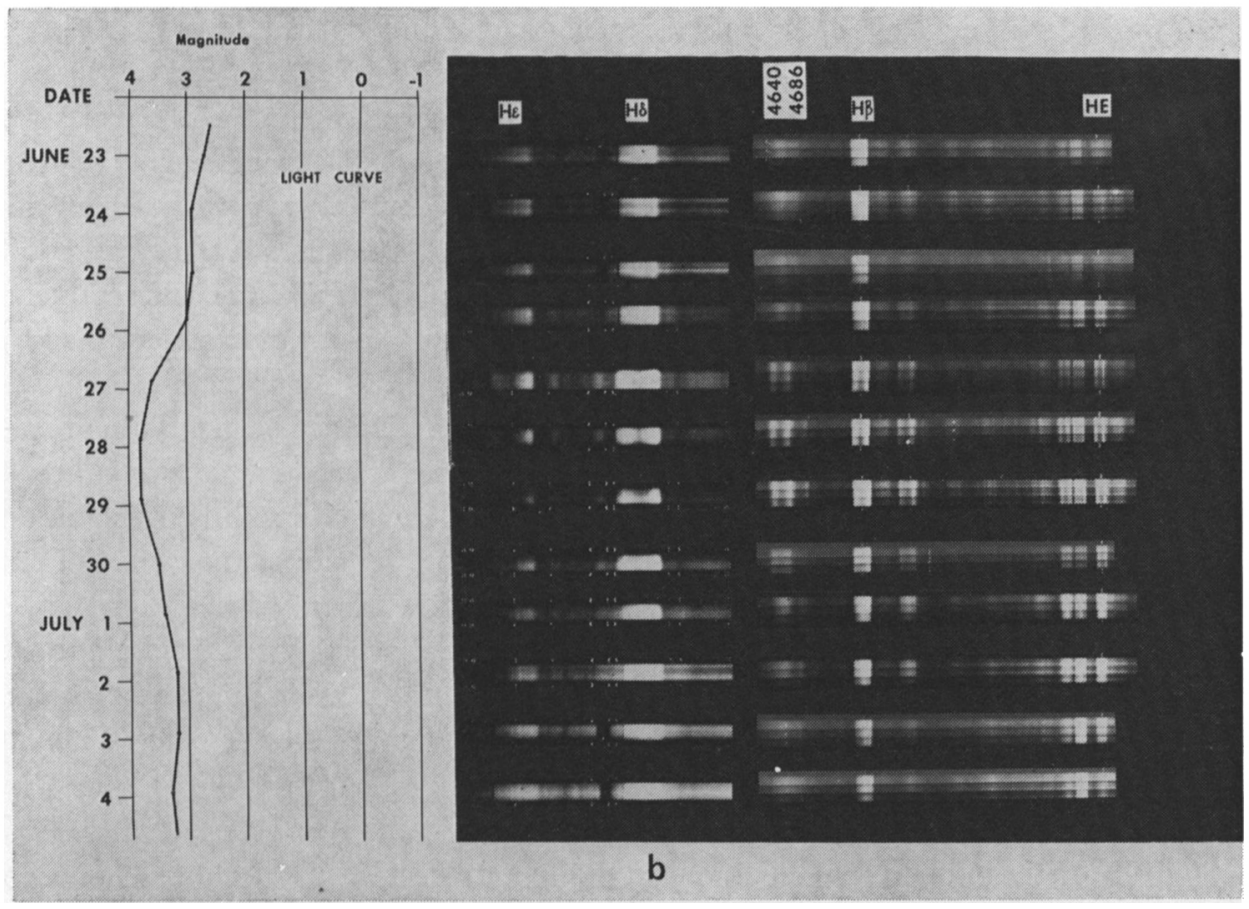


difference shows in the totally different velocity structure of lines originating in the two objects.

Figure 14a is a further attempt to provide a comprehensive view of the spectral development of V603 Aql.

(a) The basic shell structure shows clearly in hydrogen and other lines with welldefined edges and shortward absorptions.

(b) The elements $\mathrm{He}, \mathrm{N}, \mathrm{O}, \ldots$ which characterize the nova are also present in the shell. Lines arising from these elements are mixtures of shell lines and nova lines. $\mathrm{He}$ II at $\lambda 4684$ and NIII at $\lambda 4640$ are excellent examples. Note that the equatorial part of the shell has a clear counterpart in the lines, but there are no definite edges to the lines which extend to very large velocities characterizing the outflow of gas from the nova.

(c) With the appearance of the double absorption lines in the shell, there also appeared the $\mathrm{N}$ in doublet at rest wavelengths 4097 and $4103 \AA$. These lines arise from the Bowen $\mathrm{He}-\mathrm{O}-\mathrm{N}$ fluorescence process which was strong in the ejecta of the nova. The presence of this $\mathbf{N}$ III doublet always indicated gas flow from the nova.

Figure $14 \mathrm{~b}$ continues the time sequence of spectra and covers the first light fluctuation. The sequence of events accompanying fading light in a fluctuation were as follows.

(a) Flaring stopped; gas outflow from the nova ceased. The $\mathrm{N}$ III doublet at $\lambda 4097$ and $\lambda 4103$ arising from Bowen fluorescence increased in velocity and faded away.

(b) At minimum light all lines exhibited well-defined edges. Hazy lines associated with nova gas flow disappeared. Only shell lines were visible. The lines at $\lambda \lambda 4684$ and 4640 showed well defined edges; only the shell components of these lines were visible during the minimum of a light fluctuation.

(c) At and near minimum light of a fluctuation the central sections of the shell lines - those sections arising from the equatorial region of the shell - faded rapidly relative to the portions of the lines arising from the mid-latitude sections of the shell. With decline of the nova activity and cessation of gas flow from the nova the source of excitation of the shell was cut off. Different electron densities existed in different parts of the shell; intensities in the different parts declined at different rates determined by the electron density. The electron density in the equatorial region of the shell was $10^{7}-10^{8} \mathrm{~cm}^{-3}$; the decay constant for the hydrogen and other elements there was therefore less than $24 \mathrm{~h}$, in agreement with observation.

The situation for the forbidden lines was quite different because their excitation process is different. The decay constant for [N II] $\lambda .5755$ was much longer; it did not fade as did the hydrogen.

The changes in the $\mathrm{N} 1$ and $\mathrm{N} 2$ lines of [O III] were especially interesting, but the time allocated to this review does not allow discussion of their intensity changes, which were also controlled by the electron density and temperature present in different parts of the shell.

(d) As the nova brightened and started ejecting gas again, the $\mathrm{N}$ III pair at $\lambda \lambda 4097$ and 4103 first reappeared at high velocity; they grew in strength as their velocity 
decreased. The various changes discussed earlier reversed; $\lambda \lambda 4686$ and 4640 became hazy.

This entire process repeated each time a light fluctuation occurred.

\section{A Physical Model of the Nova}

Figure 15 presents a model of V603 Aql that brings all of these diverse phenomena together. The model is based largely on the important fundamental worker of Walker $(1954,1956)$ and of Kraft $(1959,1964)$ on Nova DQ Her.

V603 Aql is a binary consisting of a late dwarf that fills its inner Lagrangian surface and a hydrogen exhausted white dwarf that is the nova. The chemical composition of the late dwarf is normal. The observed binary period of $\mathrm{V} 603$ is $3^{\mathrm{h}} 20^{\mathrm{m}}$. The observed radial velocity variation is small; the tilt of the axis of rotation relative to the direction towards the Sun is therefore small as shown on the diagram.

The masses of the nova and the dwarf are equal, $\sim 0.25 M_{\odot}$.

The diameter of the nova is $\sim 10^{8} \mathrm{~cm}$; the diameter of the inner Lagrangian surface of the dwarf is $\sim 3 \times 10^{10} \mathrm{~cm}$.

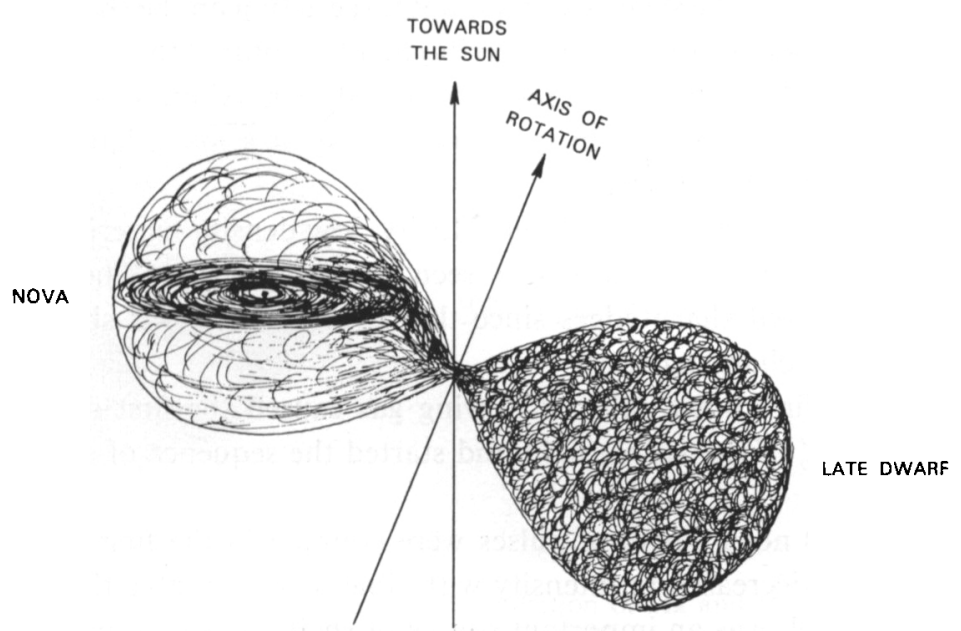

Fig. 15. Model of V603 Aql before the nova outburst.

Gas of normal chemical composition flows from the late dwarf into the inner Lagrangian surface of the nova. A disk of gas forms around the nova. The electron density in the disk is $\sim 3 \times 10^{14} \mathrm{~cm}^{-3}$; its temperature is $\sim 4 \times 10^{4} \mathrm{~K}$. Under normal conditions before the nova explosion, most of the light of the binary pair comes from this disk, which has a thickness of $10^{7}$ or a few $\times 10^{7} \mathrm{~cm}$.

The nova process starts, caused, possibly, by an excess of hydrogen from the disk mixing with the material of the hydrogen-exhausted white dwarf. The exploding gas encounters the disk, piling up against it and being forced to flow off in conicallyshaped streams. Density irregularities in the radial structure of the disk will be 
transformed into latitude irregularities in the outflowing gas. These remarks are based on simple essentially intuitive calculations. A problem of the type encountered here appears never to have been solved by the aerodynamicists. It needs to be investigated.

The expanding gas from the nova thus sweeps up the disk, hence, as suggested by Sparks and Starrfield (1973), expansion is slowed in the equatorial region. The shell becomes prolate rather than spherical. The equatorial region of the shell has the highest density; it consists largely of the old swept-up disk.

The nova shell is formed largely, probably almost entirely, from gas which surrounded the nova and originally came from the dwarf. Its chemical composition is therefore essentially normal. The gas that surrounded the nova must have been swept up in the explosive outflow in a matter of minutes. At the velocity of $1000 \mathrm{~km}^{-1} \mathrm{~s}$ the gas passed beyond the edge of the disk in $100 \mathrm{~s}$.

After the first explosive ejection, the gaseous outflow from the nova was pulsed in a period of $\sim 10$ days. These pulses caused the light fluctuations during the transition stage. The first pulse started at or shortly before maximum light. Gas was ejected from the nova at several times shell expansion velocity. The outflowing gas, which was not isotropic in distribution, impinged on the shell, causing the pressure that accelerated and distorted the shell. The resultant force acting on the shell (at maximum, 3 to 4 orders of magnitude greater than gravity) finally ruptured the shell at two places, causing the double absorption lines seen shortly after maximum light and creating the holes in the shell that were seen spectroscopically at a much later time after the shell had expanded to visible size.

The gas flow from the nova, very high velocity, low density material, provided the source of the broad, hazy, edgeless lines seen in the spectrum. The shell lines, on the other hand, showed sharp edges since they arose from a gas shell of compact, well-defined velocity characteristics.

Cessation of the first nova pulse of flowing gas caused the first decrease of light amounting to $\sim 50 \%$ of the visible light and started the sequence of spectral changes described earlier.

Approximately 10 nova emission pulses were counted in the first 116 days of the nova lifetime. They decreased in intensity with time. It is porbable that the nova gas impinging on the shell was an important source of shell excitation, particularly when the shell was of relatively small radius.

Time does not permit specification of further details of the model. The model does, however, account for all the spectral phenomena observed. It also accounts for the shape of the shell which bulges outward in the regions of the holes. It provides a logical explanation of the observed holes in the shell. The model offers a possible explanation of the ejected truncated cones forming the shell, but here a detailed hydrodynamical calculation needs to be made.

\section{Generality of the Model}

The shell of V603 Aql can be reconstructed in very great detail from the early spectra. 
It is a very complex structure, but it can be accounted for on the basis of a rather simple model. Is V603 unique? Not at all. It is likely that all novae are similar to what has been described here. Mustel and Boyarchuk (1970) have long pointed out the equatorial ring and polar cap structure of the shell of DQ Her. Hutchings (1972) has devised cone and ring models for N. Delphini 1967, N. Velpeculae 1968 (1), and N. Serpentis 1970. Molakpur (1973) has also proposed a model for N. Delphini consisting of polar caps and equatorial rings. This type of structure appears to be the general case for novae.

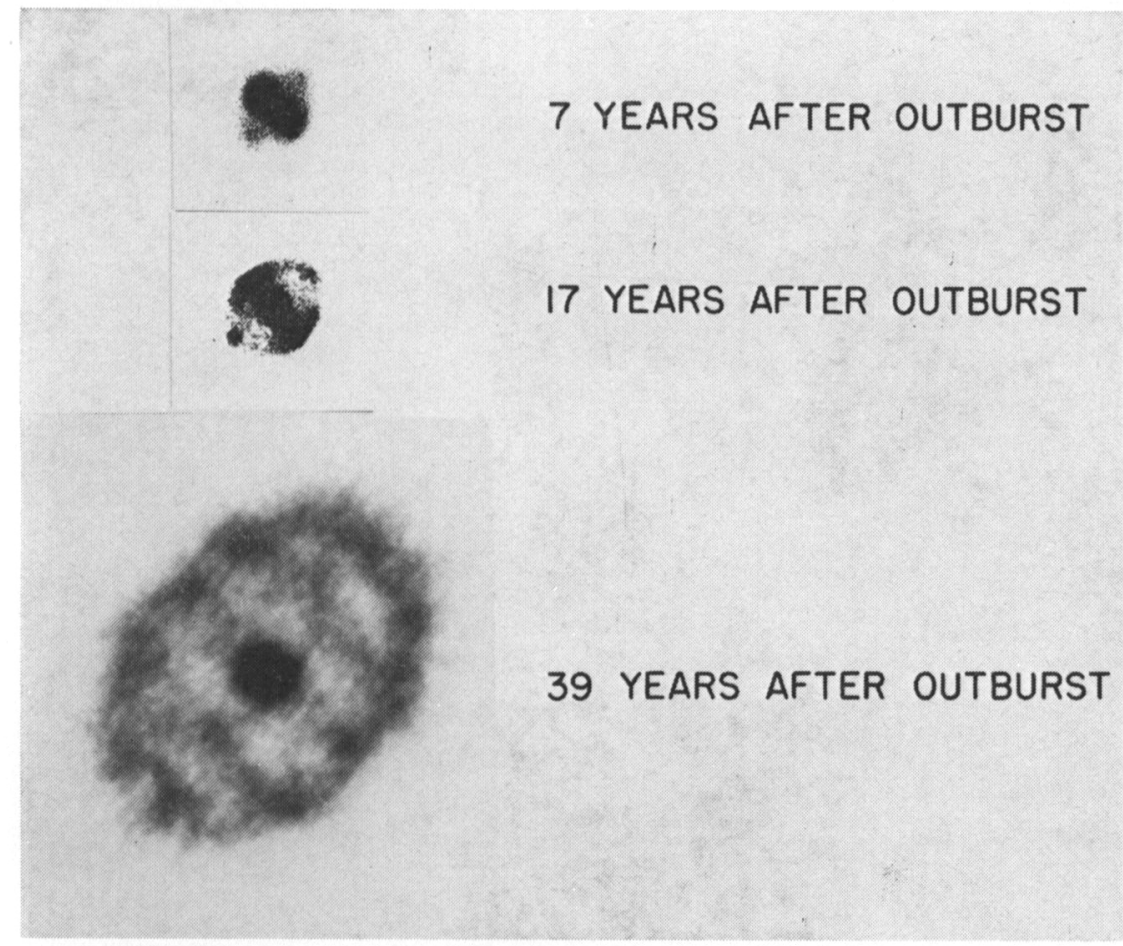

Fig. 16. Direct photographs of DQ Her in the radiation of $\mathrm{Ha}$ and [N II]. The pictures in 1942 and 1951 were taken by W. Baade. The picture from 1973 was taken by Ford and Jenner. I am grateful to them for permission to show this remarkable photograph. The three pictures are not all to the same scale; that is, the ratio of image size to shell diameter is not the same for all three pictures.

Finally, in Figure 16 I show two early photographs of Nova DQ Her taken by Baade in 1942 and 1951 along with a most remarkable photograph of DQ Her taken in May 1973 by Ford and Jenner with the $120^{\prime \prime}$ at the Lick Observatory. I am grateful to them for permitting me to show this picture.

From these photographs, particularly the remarkable one by Ford and Jenner, there can be no doubt that a nova shell is a most remarkable geometrical structure having a very high order of spatial symmetry. 


\section{References}

Barnard, E. E.: 1919, Astrophys. J. 49, 199.

Barnard, E. E.: 1920, Monthly Notices Roy. Astron. Soc. 80, 582.

Hutchings, J. B.: 1972, Monthly Notices Roy. Astron. Soc. 158, 177.

Kraft, R. P.: 1959, Astrophys. J. 130, 110.

Kraft, R. P.: 1964, Astrophys. J. 139, 469.

Malakpur, I.: 1973, Astron. Astrophys. 24, 125.

Mustel, E. R. and Boyarchuk, A. A.: 1970, Astrophys. Space Sci. 6, 183.

Sparks, W. M. and Starrfield, S. G.: 1973, Monthly Notices Roy. Astron. Soc., in press.

Walker, M.: 1954, Publ. Astron. Soc. Pacific. 66, 230.

Walker, M.: 1956, Astrophys. J. 123, 68.

Wright, W. H.: 1919, Lick Obs. Bull. 10, 30. 